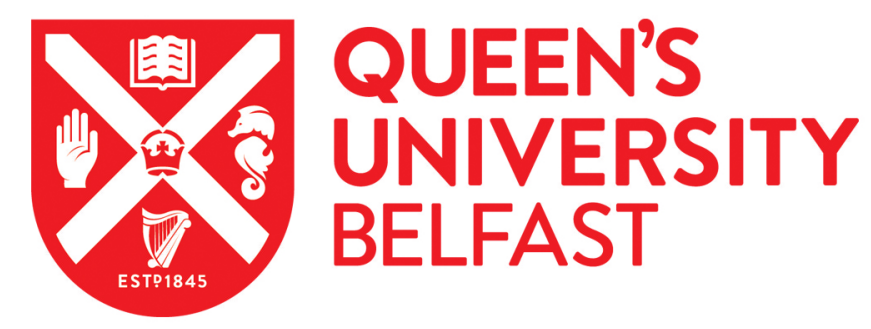

\title{
Wave Digital Filter Modeling of Circuits with Operational Amplifiers
}

Werner, K. J., Dunkel, W. R., Rest, M., Olsen, M. J., \& Smith, J. O. (2016). Wave Digital Filter Modeling of Circuits with Operational Amplifiers. In Proceedings of the 2016 24th Signal Processing Conference (EUSIPCO) (pp. 1033-1037). Institute of Electrical and Electronics Engineers Inc..

https://doi.org/10.1109/EUSIPCO.2016.7760405

Published in:

Proceedings of the 2016 24th Signal Processing Conference (EUSIPCO)

Document Version:

Publisher's PDF, also known as Version of record

Queen's University Belfast - Research Portal:

Link to publication record in Queen's University Belfast Research Portal

\section{Publisher rights}

( 2016 IEEE. Personal use of this material is permitted. Permission from IEEE must be obtained for all other uses, in any current or future media, including reprinting/republishing this material for advertising or promotional purposes, creating new collective works, for resale or redistribution to servers or lists, or reuse of any copyrighted component of this work in other works.

\section{General rights}

Copyright for the publications made accessible via the Queen's University Belfast Research Portal is retained by the author(s) and / or other copyright owners and it is a condition of accessing these publications that users recognise and abide by the legal requirements associated with these rights.

Take down policy

The Research Portal is Queen's institutional repository that provides access to Queen's research output. Every effort has been made to ensure that content in the Research Portal does not infringe any person's rights, or applicable UK laws. If you discover content in the Research Portal that you believe breaches copyright or violates any law, please contact openaccess@qub.ac.uk. 


\title{
Wave Digital Filter Modeling of Circuits with Operational Amplifiers
}

\author{
Kurt James Werner*, W. Ross Dunkel*, Maximilian Rest ${ }^{\dagger *}$, Michael Jørgen Olsen*, and Julius O. Smith III* \\ ${ }^{*}$ Center for Computer Research in Music and Acoustics, Stanford University, 660 Lomita Drive, Stanford, CA 94305, USA \\ ${ }^{\dagger}$ Fakultät Elektrotechnik und Informatik, Technische Universität Berlin, Straße des 17. Juni 135, 10623 Berlin, Germany \\ Email: [kwerner, chigi22, mrest, mjolsen, jos]@ccrma.stanford.edu
}

\begin{abstract}
We extend the Wave Digital Filter (WDF) approach to simulate reference circuits that involve operational amplifiers (op-amps). We handle both nullor-based ideal op-amp models and controlled-source-based linear op-amp macromodels in circuits with arbitrary topologies using recent derivations for complicated scattering matrices. The presented methods greatly increase the class of appropriate circuits for virtual analog modeling, and readily extend to circuits with any number of op-amps. Although op-amps are essential to many circuits and deviations from ideal can be important, previous WDF research applies only to the limited case of circuits with ideal op-amps, in differential amplifier topology, with no global feedback.
\end{abstract}

\section{INTRODUCTION}

Wave Digital Filters (WDFs) [1] are a popular approach to virtual analog modeling of audio circuitry [2] that involves combining digital models of individual circuit elements and their connection topologies into a network representing the entire circuit. Models of common circuit elements (voltage/current sources, linear one-ports, transformers, etc.) and basic connection topologies (series/parallel) have been known since the early days of WDF [1] but some elements have not been represented in the wave domain. In this paper we consider WDF modeling of a fundamental building block of electronic circuit design [3], [4]: the operational amplifier (op-amp).

Op-amps show up in relevant audio circuits including drum machines [5]-[8], guitar effect pedals [9]-[15], preamplifiers [16], and musical filters [17]. Previously, WDF methods were limited to ideal op-amps in differential amplifier configurations [11], [14] —an approach that is intractable for multiple op-amps with global feedback. In practice, op-amp circuits usually have complicated topologies and non-ideal characteristics of real op-amps can be significant [18].

In this paper we illustrate how topological techniques introduced in [13] enable WDF simulation of circuits with an arbitrary number of op-amps, modeled as ideal or non-ideal, in any topology. The paper is structured as follows: $\S \S$ II-III review op-amps and previous work on deriving WDF scattering matrices, §IV presents case studies on WDF simulations of a Bridged-T Resonator with ideal and non-ideal op-amp models, and $\S \S \mathrm{V}-\mathrm{VI}$ discuss results, future work, and conclusions.

\section{Operational Amplifiers}

Op-amps (Fig. 1a) are active, high-gain electronic devices that amplify the voltage difference between their input terminals. Modern op-amps are complex on the device level and

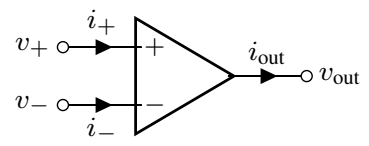

(a) Op-amp symbol.

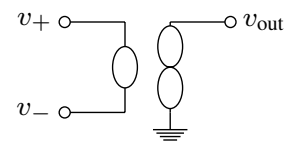

(b) Nullor-based ideal op-amp.

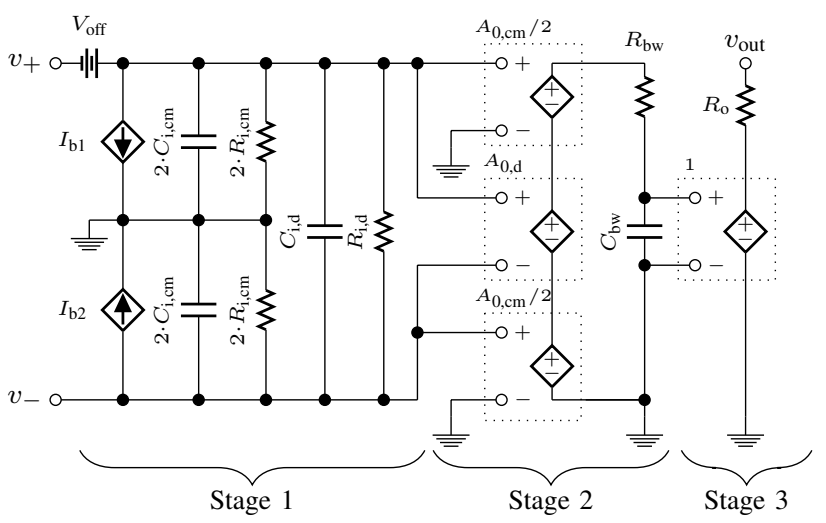

(c) A non-ideal linear op-amp macromodel [4].

Fig. 1. The op-amp, an ideal model, and a non-ideal model.

include potentially dozens of transistors [19]. To simplify circuit design, analysis, and simulation, op-amp behavior is often idealized completely or approximated using macromodels.

\section{A. Ideal Op-Amps}

An ideal op-amp has infinite open-loop gain, bandwidth, and input impedance, along with zero common-mode gain and output impedance [4]. In negative feedback, its output terminal floats to whatever voltage is necessary and sources/sinks whatever current is necessary to maintain equal input terminal voltage $\left(v_{+}=v_{-}\right)$, which neither sink nor source any current $\left(i_{+}=i_{-}=0\right)$.

Op-amps in this configuration can be represented by the network-theoretic two-port element known as the nullor with one terminal of its output port grounded (Fig. 1b). A nullor is composed of the two degenerate one-port elements known as the nullator and norator [20] (Fig. 2). The nullator is characterized by zero port voltage and zero port current, and the norator by arbitrary port voltage and current. The port between the input terminals of an ideal op-amp in negative feedback is equivalent to a nullator-its zero port voltage 


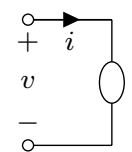

(a) nullator

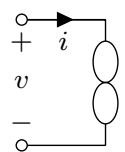

(b) norator

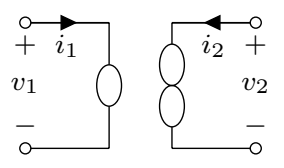

(c) nullor
Fig. 2. Symbols and port definitions for nullator, norator, and nullor.

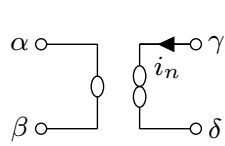

(a)

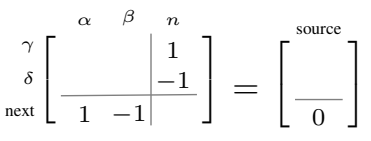

(b)
Fig. 3. Nullor (a) and element stamp (b) for Modified Nodal Analysis.

and current embody the restrictions on the op-amp's input terminals. The port between output terminal and ground is equivalent to a norator-its arbitrary port voltage and current embody the ideal op-amp's infinite gain and zero output impedance [4].

\section{B. Op-Amp Macromodels}

Op-amp macromodeling was developed in the 1970s as a reduced-complexity alternative to device-level op-amp models [21]. Macromodels are derived from device-level models using two techniques: simplification and build-up. Simplification involves replacing complicated arrangements of circuit elements with ideal elements; build-up involves the addition of circuit elements to capture some characteristics of the opamp's behavior, without necessarily resembling the devicelevel circuitry [22].

In this paper we use a three-stage linear macromodel [4] (Fig. 1c). The first stage models input effects: input offset voltage $V_{\text {off }}$, input bias currents $I_{\mathrm{b} 1}, I_{\mathrm{b} 2}$, input offset current $I_{\text {off }}$, and common mode $\left(R_{\mathrm{i}, \mathrm{cm}}, C_{\mathrm{i}, \mathrm{cm}}\right)$ and differential $\left(R_{\mathrm{i}, \mathrm{d}}\right.$, $\left.C_{\mathrm{i}, \mathrm{d}}\right)$ input impedances. The second stage models the op-amp's differential $\left(A_{0, \mathrm{~d}}\right)$ and common-mode $\left(A_{0, \mathrm{~cm}}\right)$ gains using voltage-controlled voltage sources (VCVSs) and its dominant pole / gain-bandwidth product (GBWP) via $R_{\mathrm{bw}}$ and $C_{\mathrm{bw}}$. The third stage consists of a unity-gain VCVS output buffer and an output resistance $R_{0}$. Most macromodel parameters can be extracted directly from op-amp datasheets [4] or derived from these values according to:

$$
\begin{gathered}
A_{0, \mathrm{~cm}}=\frac{A_{0, \mathrm{~d}}}{10_{\mathrm{CMRR} / 20}}, \quad f_{\mathrm{bw}}=\frac{\mathrm{GBWP}}{A_{0, \mathrm{~d}}}, \quad C_{\mathrm{bw}} R_{\mathrm{bw}}=\frac{1}{2 \pi f_{\mathrm{bw}}}, \\
I_{\mathrm{b} 1}=I_{\mathrm{b}}+I_{\mathrm{os}} / 2, \quad I_{\mathrm{b} 2}=I_{\mathrm{b}}-I_{\mathrm{os}} / 2 .
\end{gathered}
$$

Input/output impedances usually must be approximated [23].

\section{COMPlEX WDF TOPOLOGIES}

Many circuits have complex $\mathcal{R}$-type topologies that cannot be decomposed entirely into series and parallel connections. This is especially true for circuits with multiport linear elements such as transformers, controlled sources, or nullors, and is always true for circuits with at least one op-amp with feedback, no matter how it is modeled. The class of $\mathcal{R}$-type

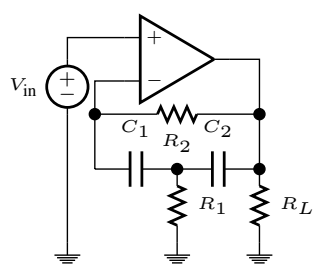

(a) Original schematic.

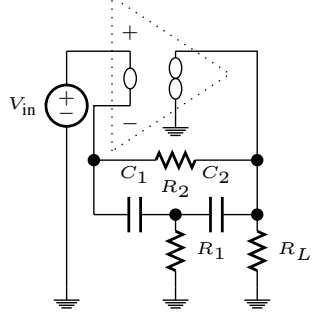

(b) Op-amp $\longrightarrow$ nullor.

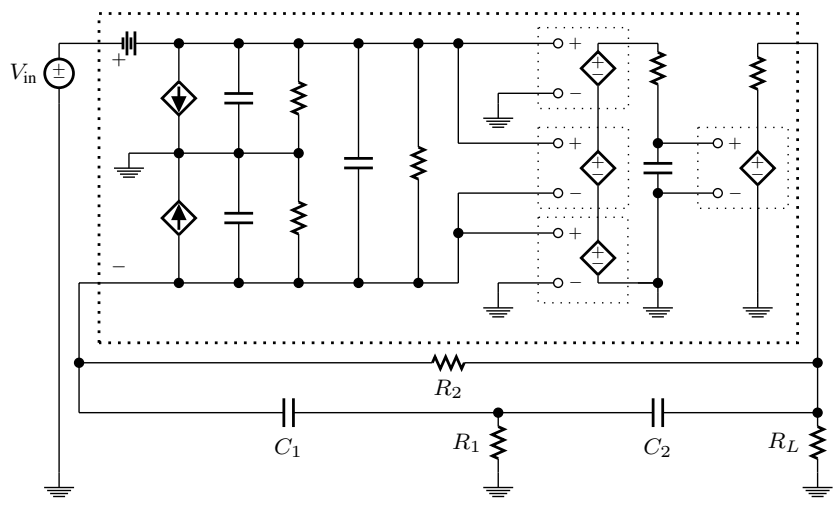

(c) Op-amp $\longrightarrow$ linear macromodel.

Fig. 4. (a) Op-amp-based Bridged-T Resonator schematic; (b) op-amp symbol replaced by nullor; and (c) op-amp symbol replaced by linear macromodel.

topologies with or without absorbed multiport linear elements is infinitely large. To form a WDF involving one of these topologies one must derive its scattering behavior. [13] gives a general procedure, which we review briefly, emphasizing cases with currents which are not port currents (involving, e.g., VCVSs and nullors) and how to handle nullor stamps.

An $\mathcal{R}$-type adaptor is characterized by the scattering among incident $(\boldsymbol{a})$ and reflected $(\boldsymbol{b})$ wave vectors, which are linear combinations of port current $\boldsymbol{i}$ and voltage $\boldsymbol{v}$ :

$$
a=v+\mathbf{R i}, \quad b=v-\mathbf{R i},
$$

where $\mathbf{R}$ is a diagonal matrix of its port resistances. $\boldsymbol{a}$ and $\boldsymbol{b}$ are related by a scattering matrix $\mathbf{S}$ [13],

$$
\boldsymbol{b}=\mathbf{S} \boldsymbol{a}, \mathbf{S}=\mathbf{I}+2\left[\begin{array}{lll}
\mathbf{0} & \mathbf{R} & \mathbf{0}
\end{array}\right] \mathbf{X}^{-1}\left[\begin{array}{lll}
\mathbf{0} & \mathbf{I} & \mathbf{0}
\end{array}\right]^{\top},
$$

where $\mathbf{I}$ is the identity matrix. $\mathbf{X}$ is a Modified Nodal Analysis (MNA) matrix characterizing the $\mathcal{R}$-type adaptor with attached instantaneous Thévenin port equivalents [13]. $\mathbf{X}$ is formed using element stamp methods [24] which embody Kirchhoff's Current Law and branch relationships. "Stamping" in voltage sources corresponding to Thévenin equivalents before multiport linear elements with branch currents (e.g., VCVSs and nullors) gives an MNA system for finding $\mathbf{S}$ with the form:

$$
\underbrace{\left[\begin{array}{ccc}
\mathbf{Y} & \mathbf{A}_{1} & \mathbf{A}_{2} \\
\mathbf{B}_{1} & \mathbf{D}_{11} & \mathbf{D}_{12} \\
\mathbf{B}_{2} & \mathbf{D}_{21} & \mathbf{D}_{22}
\end{array}\right]}_{\mathbf{X} \text { matrix }}\left[\begin{array}{l}
\boldsymbol{v}_{n} \\
\boldsymbol{j}_{1} \\
\boldsymbol{j}_{2}
\end{array}\right]=\left[\begin{array}{l}
\mathbf{0} \\
\boldsymbol{e} \\
\mathbf{0}
\end{array}\right],
$$

where the partitions of $\mathbf{X}$ define the relationships among node voltages $\boldsymbol{v}_{n}$, voltage source branch currents $\boldsymbol{j}_{1}=-\boldsymbol{i}$, other branch currents $\boldsymbol{j}_{2}$, and voltage source values $\boldsymbol{e}=\boldsymbol{a}$. 


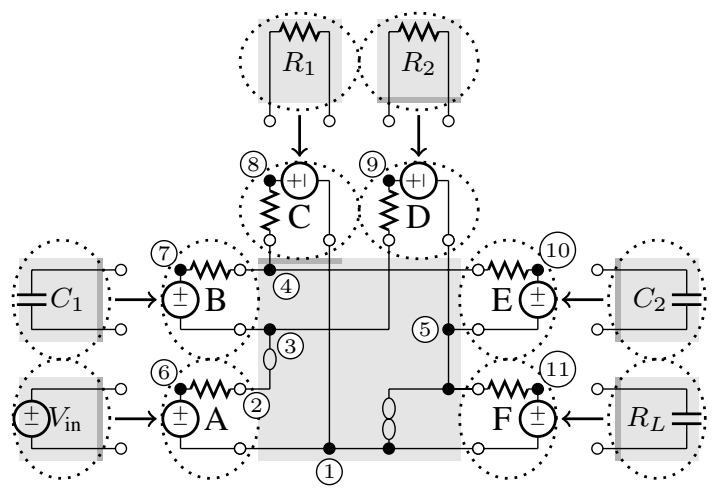

(a) Nullor-based Bridged-T Resonator.

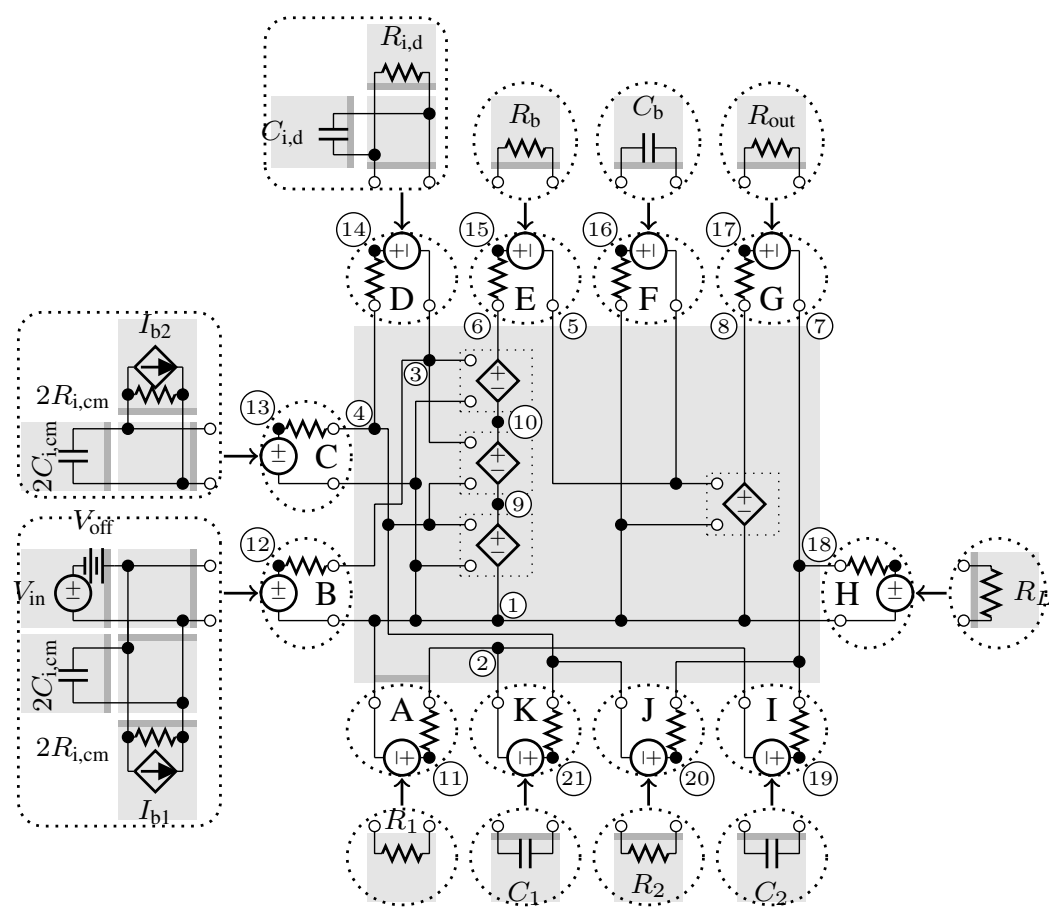

(b) Macromodel-based Bridged-T Resonator.

Fig. 5. Reference circuits rearranged to highlight WDF adaptor structures corresponding to (a) the nullor-based model (Fig. 4b); and (b) the macromodel-based model (Fig. 4c). WDF adaptors are represnted by shaded boxes, whose darker shaded edges indicate the adapted port as in [2]. $\mathcal{R}$-type adaptors in each are shown with Thévenin equivalents $(A \cdots F$ and $A \cdots K$, respectively) and node labels necessary for their scattering matrix derivations.

Stamps for standard circuit elements are given in literature [24], [25] - here we draw special attention to the nullor stamp (Fig. 3). This stamp is a direct expression of the properties of the nullor-the row "next" corresponds to the equality of the input voltages $\left(v_{\alpha}-v_{\beta}=0\right)$ and column " $n$ " corresponds to the arbitrary output current $\left(i_{\gamma}=-i_{\delta}\right)$.

An alternative to incorporating the behavior of nullors as element stamps is to perform row and column operations on $\mathbf{X}$ [25]. This method complicates bookkeeping of (4), so we recommend the element stamp method for finding $\mathbf{S}$.

\section{CASE STUdY: BRIDGED-T RESONATOR}

As a case study, we form WDF simulations of a representative analog drum machine circuit: the Bridged-T Resonator (Fig. 4a), a bridged-T network $\left(R_{1}, R_{2}, C_{1}, C_{2}\right)$ in the negative feedback path of an op-amp. With an ideal op-amp, it is a bandpass filter with center frequency $f_{c}$ and quality factor $Q$ :

$$
f_{c}=\frac{1}{2 \pi \sqrt{R_{1} R_{2} C_{1} C_{2}}}, \quad Q=\frac{\sqrt{R_{2} / R_{1}}}{\sqrt{C_{1} / C_{2}}+\sqrt{C_{2} / C_{1}}} .
$$

We add a representative load resistor $R_{L}$ on the output and assume that $V_{\text {in }}$ has $1 \Omega$ of source resistance.

The Bridged-T Resonator "rings" in response to impulsive signals, creating a decaying sinusoid of frequency $f_{c}$ whose decay rate depends on $Q$. Classic analog drum machines like the Roland TR-808 [26], TR-606, TR-909, CR-5000/8000, etc. use variations on the Bridged-T Resonator in voice circuits including bass drums [5], toms, congas, claves, rimshots, cymbals, and hi-hats [6].

\section{A. Nullor-Based Model}

To form a nullor-based WDF model of the Bridged-T Resonator, we first replace the op-amp in Fig. 4a with a nullor, yielding Fig. 4b. As in [27], we form a graph with circuit nodes as nodes, one-ports as edges, and replacement graphs for the nullor (a multiport linear element); perform a search for "split components"; and derive a WDF adaptor structure (Fig. 5a) from the resulting SPQR tree.

Most of the elements in this WDF adaptor structure (resistive voltage source, resistors, capacitors) have been known since the early days of the field. However the large $\mathcal{R}$-type adaptor, which includes the nullor, requires special treatment. Following the procedure in [13], we attach an instantaneous Thévenin equivalent to each port $A \cdots F$ (shown in Fig. 5a), use element stamps representing the nullor (Fig. 3), and Thévenin resistors and voltage sources to populate the MNA matrix $\mathbf{X}$ describing the $\mathcal{R}$-type adaptor (Fig. 6), which is then used to solve for its scattering matrix according to (3).

\section{B. Linear Macromodel}

To form a macromodel-based WDF model of the Bridged$\mathrm{T}$ Resonator, we first replace the op-amp in Fig. 4a with the linear macromodel shown in Fig. 1c, yielding Fig. 4c. Again following the procedure of [27], we find the WDF adaptor structure in Fig. $5 \mathrm{~b}$ and solve for the scattering behavior of the $\mathcal{R}$-type adaptor according to [13].

Notice that although feedback in the op-amp has caused a complicated $\mathcal{R}$-type adaptor with all four VCVSs absorbed, 


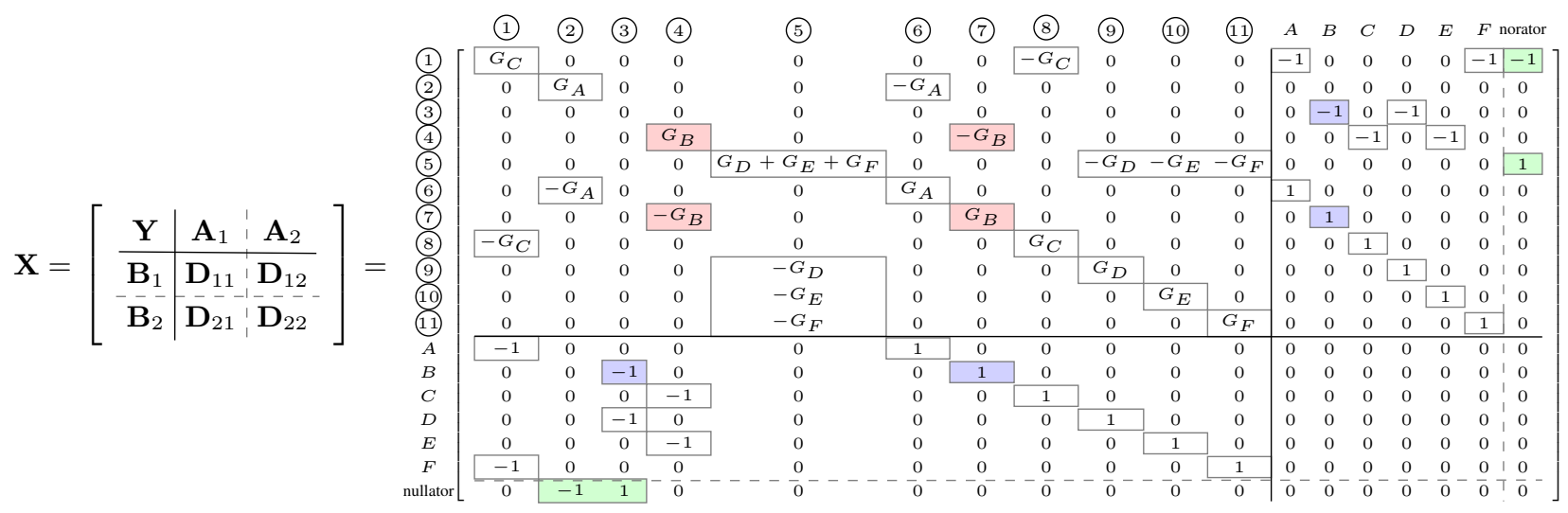

Fig. 6. Forming MNA matrix $\mathbf{X}$, highlighting examples of resistor (red, $R_{B}$ ), voltage source (blue, $e_{b}$ ), and nullor (green, Fig. 3) element stamps.

TABLE I

Simulation PARAMETERS AND RESUlts.

\begin{tabular}{|r|c|c|c|c|c|}
\hline component & $R_{1}$ & $R_{2}$ & $C_{1}$ & $C_{2}$ & $R_{L}$ \\
\hline value & $500 \Omega$ & $10 \mathrm{M} \Omega$ & $1 \mathrm{nF}$ & $1 \mathrm{nF}$ & $10 \mathrm{k} \Omega$ \\
\hline
\end{tabular}

(a) Bridged-T Resonator Component Values.

\begin{tabular}{|c|c|c|c|c|c|}
\hline & ideal & NJM2904D & MC4558L-D08 & $\mu \mathrm{A} 741 \mathrm{TC}$ & units \\
\hline$V_{\text {off }}$ & 0 & 2 & 2 & 1 & $\mathrm{mV}$ \\
\hline$I_{\mathrm{b}}$ & 0 & 25 & 30 & 80 & $\mathrm{nA}$ \\
\hline$I_{\mathrm{os}}$ & 0 & 5 & 5 & 20 & $\mathrm{nA}$ \\
\hline$A_{0, \mathrm{~d}}$ & $\infty$ & 100 & 200 & 200 & $\mathrm{~V} / \mathrm{mV}$ \\
\hline CMRR & $\infty$ & 85 & 90 & 90 & $\mathrm{~dB}$ \\
\hline GBWP & $\infty$ & 0.6 & 2.8 & 1 & $\mathrm{MHz}$ \\
\hline$R_{\mathrm{i}, \mathrm{cm}}$ & $\infty$ & 5 & 5 & 5 & $\mathrm{M} \Omega$ \\
\hline$R_{\mathrm{i}, \mathrm{d}}$ & $\infty$ & 3 & 2 & 5 & $\mathrm{M} \Omega$ \\
\hline$R_{\mathrm{o}}$ & 0 & 75 & 75 & 75 & $\Omega$ \\
\hline$C_{\mathrm{i}, \mathrm{cm}}$ & $\infty$ & 2 & 2 & 2 & $\mathrm{pF}$ \\
\hline$C_{\mathrm{i}, \mathrm{d}}$ & $\infty$ & 1.4 & 1.4 & 1.4 & $\mathrm{pF}$ \\
\hline$-\overline{A_{0, \mathrm{~cm}}}$ & 0 & $5 . \overline{6} 2 \overline{3}$ & $6.3 \overline{2} 5$ & $\overline{6.3} \overline{2} 5^{-}$ & $-\frac{1}{\mathrm{~V} / \mathrm{V}}$ \\
\hline$f_{\mathrm{bw}}$ & - & 6 & 14 & 5 & $\mathrm{~Hz}$ \\
\hline$R_{\mathrm{bw}}$ & - & 100 & 100 & 100 & $\mathrm{k} \Omega$ \\
\hline$C_{\mathrm{bw}}$ & - & 0.2653 & 0.1137 & 0.3183 & $\mu \mathrm{F}$ \\
\hline$I_{\mathrm{b} 1}$ & 0 & 27.5 & 32.5 & 90 & $\mathrm{nA}$ \\
\hline$I_{\mathrm{b} 2}$ & 0 & 22.5 & 27.5 & 70 & $\mathrm{nA}$ \\
\hline
\end{tabular}

(b) Op-Amp Parameters.

\begin{tabular}{|r|c|c|c|c|c|}
\cline { 2 - 6 } \multicolumn{1}{c|}{} & ideal & JRC2904 & $\mu$ PC4558C & $\mu$ A741TC & units \\
\hline$f_{c}$ & 2.232 & 1.805 & 2.115 & 1.945 & $\mathrm{kHz}$ \\
$Q$ & 72.074 & 38.224 & 54.595 & 53.221 & - \\
peak $(\mathrm{dB})$ & 60.000 & 52.680 & 57.124 & 56.142 & $\mathrm{~V}(\mathrm{~dB})$ \\
DC offset & 0 & -228.988 & -278.990 & -701.986 & $\mathrm{mV}$ \\
\hline
\end{tabular}

(c) Simulation Results Summary.

the $\mathcal{R}$-type adaptor is not equivalent to the the op-amp in any sense-it is a consequence of the topology of the entire circuit, and electrical elements from "inside" the op-amp end up pulled out of the $\mathcal{R}$-type adaptor where appropriate (e.g., the series combination of $V_{\text {off }}$ and $V_{\text {in }}$ and the parallel combinations of $2 \cdot R_{\mathrm{i}, \mathrm{cm}}, 2 \cdot C_{\mathrm{i}, \mathrm{cm}}, I_{\mathrm{b} 1}$ and $\left.I_{\mathrm{b} 2}\right)$.

\section{Results \& Discussion}

We ran simulations using the representative (compare against [26]) component values given in Table Ia for both the ideal nullor-based model ( $(\mathrm{IV}-\mathrm{A})$ and the linear macromodel (§IV-B). Both took a $10-\mathrm{mV}$ impulse as input. The macro- model simulation was run with the parameter values shown in Table Ib, which were extracted from the datasheets of three common audio op-amps: the NJM2904D [28], the MC4558LD08 [29], and the $\mu$ A741TC [30]. Shaded table entries were not specified on the datasheets and were either duplicated from another model or based on standard values [23]. Entries derived according to (1) are given below the dotted line.

Fig. 7 shows simulation results in the frequency domain and Table Ic summarizes extracted parameters. Accounting for the warping introduced by the bilinear transform [31], [32], $f_{c}$ and $Q$ for the ideal simulation closely match the values predicted by (5). The macromodel simulations diverge from ideal behavior, including audible differences in frequency and decay time.

The scattering $\boldsymbol{b}=\mathbf{S} \boldsymbol{a}$ for the $\mathcal{R}$-type adaptor in Fig. 5a is

$\left[\begin{array}{l}b_{A} \\ b_{B} \\ b_{C} \\ b_{D} \\ b_{E} \\ b_{F}\end{array}\right]=\left[\begin{array}{cccccc}1 & 0 & 0 & 0 & 0 & 0 \\ -1.000 & -0.001 & -1.000 & 0.001 & -0.001 & 0 \\ -1.000 & -0.999 & 0 & -0.001 & 0.001 & 0 \\ 882.000 & 882.998 & 882.000 & 0.002 & 0.998 & 0 \\ 883.000 & 881.999 & 883.000 & 1.001 & -0.001 & 0 \\ 884.000 & 882.998 & 882.000 & 1.002 & 0.998 & -1\end{array}\right]\left[\begin{array}{l}a_{A} \\ a_{B} \\ a_{C} \\ a_{D} \\ a_{E} \\ a_{F}\end{array}\right](6)$

where entries of $\mathbf{S}$ are denoted as $s_{m n}, m, n \in[A \cdots F]$, meaning the contribution of $a_{n}$ to $b_{m}$. For example, $s_{F A}=$ 884.00 .

$s_{A A}=1$ indicates perfect reflection off the ideal op-amp's infinite input impedance. The zeros in the rest of the row signify that waves incident on other ports don't contribute to outgoing waves at port $A$. Similarly, the $s_{F F}=-1$ indicates perfect inverting reflection off of the ideal op-amp's zero output impedance and the zeros in the rest of the column indicate that waves incident on port $F$ don't contribute to outgoing waves at any other ports.

For adaptors without absorbed nullors, every entry in $\mathbf{S}$ will depend on all port resistances $R_{A} \cdots R_{F}$. Tuning the upwardfacing port resistance (here, $R_{C}$ ) so that its diagonal entry in $\mathbf{S}$ is zero (here, $s_{C C}=0$ ) is called "adapting" this port. However, here the elements in row $A$ and column $F$ are independent of the chosen port resistances. This means that neither port $A$ (input) nor $F$ (output) can be adapted, since neither $s_{A A}$ nor 


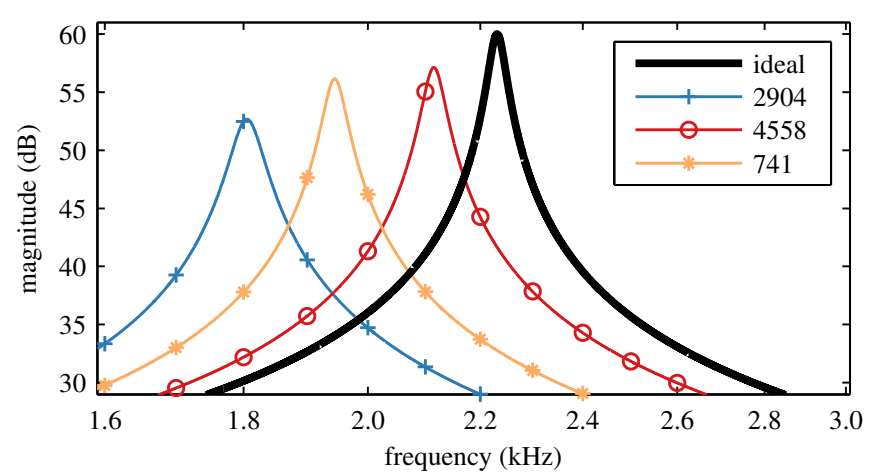

Fig. 7. Results of four simulations in the frequency domain.

$s_{F F}$ can be set to zero. In practice, this is not a problemperfect reflections actually represent an opportunity to break a WDF simulation into smaller sub-simulations [2], [11].

\section{CONClusion \& Future Work}

The approach presented in this paper enables WDF simulation of circuits involving any number of op-amps in any topology, modeled as ideal or as linear macromodels. Previously, WDFs could only handle circuits with op-amps in differential amplifier configuration, modeled as ideal [11], [14].

Choosing a suitable op-amp model for a WDF simulation involves balancing complexity and accuracy. Nullor-based ideal models have low complexity and can be sufficiently accurate; incorporating aspects of linear macromodels increases complexity but can capture important behavioral details.

Our results indicate that the ideal op-amp model and different parameterizations of a three-stage linear macromodel lead to audible differences in the Bridged-T Resonator WDF simulation. The DC offset introduced by the macromodel input bias currents $I_{\mathrm{b} 1}$ and $I_{\mathrm{b} 2}$ could have perceptually relevant effects if the resonator were embedded in a larger multi-stage or nonlinear circuit.

The op-amp is one of a wide class of amplifiers that can be modeled as nullors or linear macromodels, including operational inverting, current, floating [19], and transconductance amplifiers, and current-feedback op-amps. Beyond amplifiers, nullors can be used to model ideal transistors, and linear controlled sources can be used as part of linearized models of nonlinear devices, e.g., the Hybrid- $\pi$ model of a bipolar junction transistor [3]. The methods presented in this paper are applicable to WDF modeling of all of these devices.

Though the current work considers only linear models, nonlinear behaviors including slew-rate limiting and transfer, input, and output nonlinearities [18] are often included in opamp macromodels alongside device-level models of differential transistor input stages [21]. Future work will incorporate such nonlinear macromodels into WDF simulations, building on a recent general framework for handling multiple nonlinearities [33], [34] or non-adaptable linear elements [35] alongside complex topologies.

\section{REFERENCES}

[1] A. Fettweis, "Wave digital filters: Theory and practice," Proc. IEEE, vol. 74, pp. 270-327, 1986.

[2] G. De Sanctis and A. Sarti, "Virtual analog modeling in the wave-digital domain," IEEE Trans. Audio, Speech, Language Process., vol. 18, 2010.

[3] L. Bruton, RC-Active Circuits. Prentice-Hall Inc., 1980.

[4] A. Sedra and K. Smith, Microelectronic Circuits, 5th ed., 2004.

[5] K. Werner et al., "A physically-informed, circuit-bendable, digital model of the Roland TR-808 bass drum circuit," in Proc. Int. Conf. Digital Audio Effects (DAFx-14), Erlangen, Germany, Sept. 1-5 2014.

[6] —, "The TR-808 cymbal: a physically-informed, circuit-bendable, digital model," in Proc. Int. Comput. Music / Sound Music Comput. Conf., vol. 40/11, Athens, Greece, Sept. 14-20 2014.

[7] _ - "More cowbell: a physically-informed, circuit-bendable, digital model of the TR-808 cowbell," in Proc. 137 Conv. Int. Audio Eng. Soc. (AES), Los Angeles, CA, Oct. 9-12 2014.

[8] D. Yeh et al., "Physical and behavioral circuit modeling of the SP-12 sampler," in Proc. Int. Comput. Music Conf., Copenhagen, 2007.

[9] _ "Simplified, physically-informed models of distortion and overdrive guitar effect pedals," in Proc. Int. Conf. Digital Audio Effects (DAFx-07), Bordeaux, France, Sept. 10-15 2007.

[10] M. Holters et al., "A digital emulation of the Boss SD-1 Super Overdrive pedal based on physical modeling," in Proc. 131st Conv. Audio Eng. Soc. (AES), New York, NY, Oct. 20-23 2011.

[11] R. Paiva et al., "Emulation of operational amplifiers and diodes in audio distortion circuits," IEEE Trans. Circuits Syst. II, vol. 59, 2012

[12] F. Eichas et al., "Physical modeling of the MXR Phase 90 guitar effect pedal," in Proc. DAFx-14, 2014.

[13] K. Werner et al., "Wave digital filter adaptors for arbitrary topologies and multiport linear elements," in Proc. Int. Conf. Digital Audio Effects (DAFx-15), Trondheim, Norway, Nov. 30 - Dec. 32015.

[14] _ - "An improved and generalized diode clipper model for wave digital filters," in Proc. 139 Conv. Audio Eng. Soc. (AES), New York, NY, Oct. 29 - Nov. 12015.

[15] C. Gnegy and K. Werner, "Digitizing the Ibanez Weeping Demon wah pedal," in Proc. DAFx-15, 2015.

[16] K. Dempwolf et al., "Discretization of parametric analog circuits for real-time simulations," in Proc. Int. Conf. Digital Audio Effects (DAFx10), Granz, Austria, Sept. 6-10 2010.

[17] J. Parker and S. D'Angelo, "A digital model of the Buchla LowpassGate," in Proc. Int. Conf. Digital Audio Effects (DAFx-13), Maynooth, Ireland, Sept. 2-5, 2013.

[18] R. Gaskell, "Modeling the nonlinear behavior of operational amplifiers," in Proc. 137 Conv. Audio Eng. Soc., Los Angeles, CA, Oct. 9-12 2014.

[19] J. Huijsing, Operational Amplifiers: Theory and Design, 2011.

[20] H. Carlin, "Singular network elements," IEEE Trans. Circuit Theory, vol. 11, pp. 67-72, Mar. 1964.

[21] G. Boyle et al., "Macromodeling of integrated circuit operational amplifiers," IEEE J. of Solid-State Circuits, vol. 9, Dec. 1974.

[22] B. Cohn et al., "Macromodeling of operational amplifiers," in Proc. IEEE Int. Solid-State Circuits Conf., Feb. 13 1974, pp. 42-43.

[23] R. Mancini, Op Amps For Everyone. Texas Instruments, Sept. 2001.

[24] C.-W. Ho et al., "The modified nodal approach to network analysis," IEEE Trans. Circuits Syst, vol. 22, no. 6, pp. 504-509, 1975.

[25] J. Vlach and K. Singhal, Computer methods for circuit analysis and design. Springer, 1983.

[26] Roland Corporation, “TR-808 service notes," June 151981.

[27] D. Fränken et al., "Generation of wave digital structures for networks containing multiport elements," IEEE Trans. Circuits Syst. I: Reg. Papers, vol. 52, pp. 586-596, 2005.

[28] New Japan Radio Co., "NJM2904,” Mar. 202014.

[29] Unisonic Technologies, "MC4558 (\#QW-R105-002.H)," 2011.

[30] Texas Instruments, “ $\mu$ A741 (\#SLOS094E),” Nov. 1970 (rev. Jan. 2015).

[31] F. G. Germain and K. J. Werner, "Design principles for lumped model discretisation using Möbius transforms," in Proc. DAFx-15, 2015.

[32] J. Smith III, Physical Audio Signal Processing, 2010.

[33] K. Werner et al., "A general and explicit formulation for wave digital filters with multiple/multiport nonlinearities and complicated topologies," in Proc. IEEE WASPAA, New Paltz, NY, October 18-21 2015.

[34] — , "Resolving wave digital filters with multiple/multiport nonlinearities," in Proc. DAFx-15, 2015.

[35] - "A computational model of the Hammond organ vibrato/chorus using wave digital filters," in Proc. Int. Conf. Digital Audio Effects (DAFx-16), Brno, Czech Republic, Sept. 5-9 2016. 